\title{
Study of PVC-based Skin Phantom with graphite particles in Terahertz Frequency Range
}

\author{
T. Zhang ${ }^{1}$, M. K. Khodzitsky ${ }^{1}$, P. S. Demchenko ${ }^{1}$, \\ A. V. Bykov ${ }^{1,2}$, A. P. Popov ${ }^{1,2}$ and I. V. Meglinski ${ }^{1,2}$ \\ ${ }^{1}$ Terahertz Biomedicine Laboratory, ITMO University, Saint Petersburg, Russia, tmzhang91@ gmail.com \\ ${ }^{2}$ Biophotonics Group, University of Oulu, Oulu, Finland
}

\section{Introduction}

The development of any optical imaging system requires the use of tissue-simulating objects (phantoms) to mimic human or animal tissues for device calibration. Calibration procedures need stable materials with accurately controlled optical properties. However, such a beneficial combination is hardly possible for real biological tissues and organs: due to extraction from their natural environment their properties change over time. Therefore, the phantoms being the substitutes for real biological objects are in high demand.

Many types of phantoms have been developed for $\mathrm{THz}$ technology in early studies. For the breast cancer, phantoms that were composed of water, oil, surfactant TX151, agar, nano-diamonds and nano-onions in varying proportions have been designed to represent the optical properties of breast tissue in $\mathrm{THz}$ frequency range [1], as well as the phantoms that were composed of water, lipid and collagen [2]. Using over-the-counter soft contact as a phantom of cornea has been reported in article [3]. The phantom that is made by porcine derived gelatin with water content from $83 \%$ to $95 \%$ was tested in article [4]. All these phantoms are hydrated phantoms due to their certain water content.

Since $\mathrm{THz}$ radiation is very sensitive to the presence of water, and water evaporation affecting the measurement, a water-free phantom needs to be developed for stable and constant usage. One of the popular and established approaches for tissue phantom manufacturing utilizes PVC as a matrix material [5]. These phantoms possess high flexibility, elasticity and serve as optical analogues of biotissue in visible and NIR spectral regions. In this article, a PVC-based, water-free phantoms to mimic human skin in $\mathrm{THz}$ frequency range was examined for the first time to our knowledge.

\section{Sample Preparation}

The proposed phantoms were originally designed for the optical and NIR spectral range and used for research in the field of optical coherence tomography (OCT) [5]. It was made using a scattering agent and a matrix material. The scattering agent was chosen to be zinc oxide $(\mathrm{ZnO})$ nanoparticles with an average diameter of $0.35 \mu \mathrm{m}$ at a concentration of $12 \mathrm{mg} / \mathrm{ml}$ to generate desired optical properties. A polyvinyl chloride-plastisol (PVCP) was used as a matrix material for fabrication. Some graphite was also added into the mixture to achieve enough absorbance. PVCP, graphite and $\mathrm{ZnO}$ nanoparticles were first mixed with each other. After sonication for $15 \mathrm{~min}$, the mixture was then poured into a rectangular aluminum mold and put into an oven $\left(180{ }^{\circ} \mathrm{C}\right)$ for $30 \mathrm{~min}$. Finally, after cooling, the fabricated phantom is stored between glass slides to prevent PVC penetration into the plastic Petry dish [5]. Four phantoms with different graphite concentrations $(10 \%, 12.5 \%, 16.7 \% 21.9 \%)$ were produced.

\section{Experiment Setup and Signal Processing}

To see, whether the phantom can be used as a proper replacement of human skin for medical research in the $\mathrm{THz}$ frequency range, the $\mathrm{THz}$ optical properties of the phantom should be assessed. Terahertz time-domain spectroscopy (THz-TDS) was used for this purpose. It is the most in-use $\mathrm{THz}$ device to measure sample's optical properties in the $\mathrm{THz}$ frequency domain [6]. It measures both the amplitude and phase of the $\mathrm{THz}$ pulse that is reflected by the sample or transmits through the sample [6]. With the help of Fourier transform, optical properties, such as refractive index, absorption coefficient and/or transmission coefficient can be finally obtained [6,7].

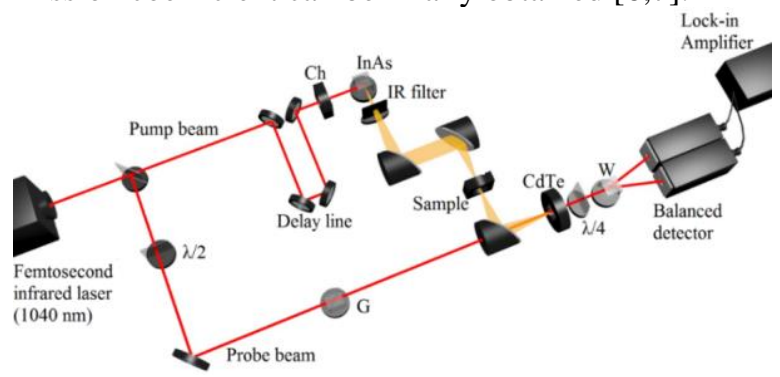

Fig. 2. The experimental setup of THz TDS system. $\lambda / 2-$ half-wave plate, $\mathrm{G}$ - Glan prism, Ch - chopper, $\lambda / 4$ - quarterwave plate, $\mathrm{W}$ - Wollaston prism

The following formulas were used to calculate the refractive index and absorption coefficient basing on the spectrums and the phases we got from the fast Fourier transform (FFT) [6,7]:

$$
\begin{gathered}
\alpha(v)=-2 \ln \left[\frac{T(v) E_{\text {sample }}(v)}{E_{\text {reference }}(v)}\right] / d \\
n(v)=1+c\left[\phi_{\text {sample }}(v)-\phi_{\text {reference }(v)}\right] /[2 \pi v d] \\
T(v)=1-R=-[n(v)-1]^{2} /[n(v)+1]^{2}
\end{gathered}
$$

where $\alpha$ - the absorption coefficient, $n$ - the refractive index of the sample, $E$ and $\phi$ - the amplitude and the phase of the signal, $d$ - the thickness of the sample, $T$ - the Fresnel loss at the air-sample interface.

\section{Results}


After the calculation using formula (1) and (2), the refractive index and absorption coefficients of the phantoms were obtained and compared with the published data [8].

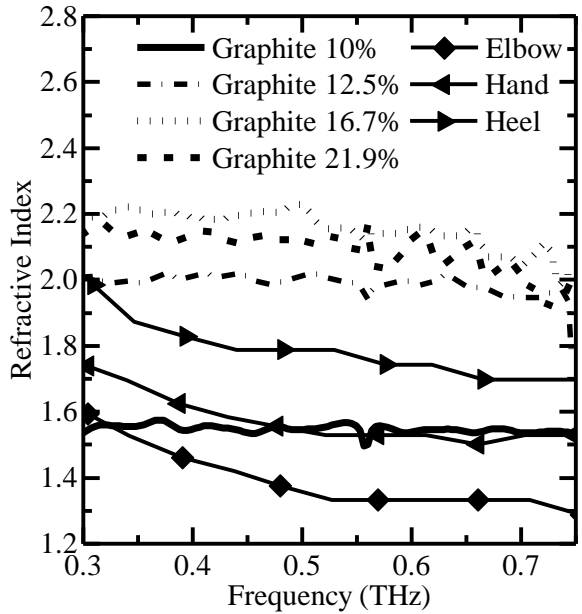

Fig. 3. Comparison of the refractive indices between phantom, elbow, hand and heel skin.

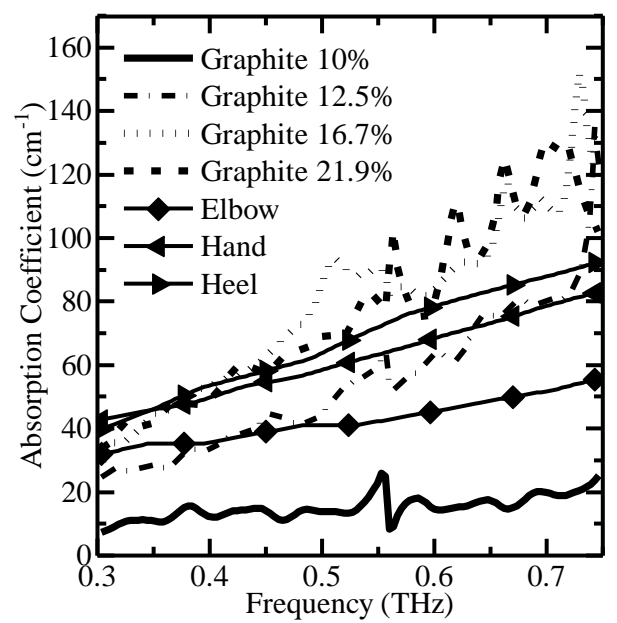

Fig. 4. Comparison of the absorption coefficients between phantom, elbow, hand and heel skin.

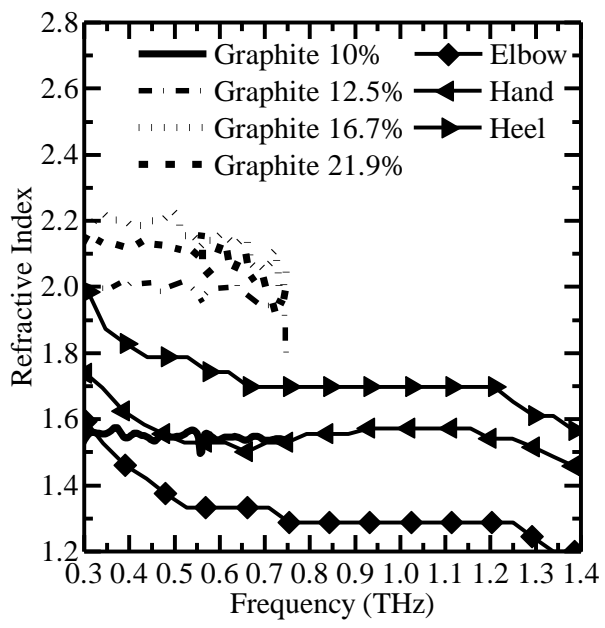

Fig. 5. Comparison of the refractive indices between phantom, elbow, hand and heel skin in a wider frequency range

Fig. 3 and Fig. 4 demonstrate the optical properties of the PVC-based phantom with graphite. The concentration of graphite influences on both refractive index and absorption coefficient of phantom. The higher the graphite concentration, the higher the value of optical properties. However, the influence of the graphite concentration also has a limit. The optical property differences between the graphite concentration of $16.7 \%$ and $21.9 \%$ are very small.

The refractive index of the phantom with $10 \%$ graphite shows good correspondence with the refractive index of hand skin in the frequency range of 0.45 $0.75 \mathrm{THz}$. Fig. 5 presents a good promise that in higher frequency range $(>0.75 \mathrm{THz})$ the phantom with $10 \%$ graphite may continue mimicking the refractive index of hand skin, since both refractive indices are nearly constant. Nonetheless, the absorption coefficient of PVC-based phantom with $10 \%$ graphite is much lower than that of any skin. The phantoms with $16.7 \%$ and $21.9 \%$ graphite possesses similar absorption coefficient as hand and heel skin in lower frequency range $(0.3-0.5 \mathrm{THz})$. The absorption coefficient of the phantom with $12.5 \%$ graphite is close to that of hand skin in higher frequency range (0.6$0.75 \mathrm{THz}$ ).

\section{Summary}

The result proves that the presented phantoms with $\mathrm{ZnO}$ nanoparticles and graphite may achieve similar optical properties as human skin. The influence of different concentrations of graphites on the optical properties is also revealed. Such characteristic gives a good promise that by choosing proper concentrations of graphite, we may achieve an ideal phantom that can mimic the optical properties of human skin. Since the phantom is water-free, it can be preserved much longer than the phantom with water or lipid while the optical properties of the phantom still maintain.

\section{Reference}

1. Bowman, T., Walter, A., et al. phantom study of terahertz spectroscopy and imaging of micro-and nano-diamonds and nano-onions as contrast agents for breast cancer // Biomed Phys Eng Express. 2007. V. 3, No. 5. P. 055001

2. Truong, B.C.Q., Fitzgerald, A.J., et al. Concentration analysis of breast tissue phantoms with terahertz spectroscopy // Biomed. Opt. Exp. 2018. V. 9. No. 3. P. 1334-1349. 3. Sung, S., Garritano, J., et al. Preliminary results of noncontact THz imaging of cornea // Proc. SPIE V. 9362. P. 93620C-1.

4. Bajwa, N., Garritano, J., et al. Reflective terahertz (THz) imaging: system calibration using hydration phantoms // Proc. SPIE. 2013. V. 8585, P. 85850W.

5. Wróbel, M. S., Popov, A. P., et al. Measurements of fundamental properties of human skin phantoms // J. Biomed. Opt., 2015. V. 20. No. 4. P. 045004

6. Dexheimer, S. L. Terahertz Spectroscopy: Principles and Applications // CRC Press. 2007.

7. Yasuda, $H$. Measurement of terahertz refractive index of metal with terahertz time-domain spectroscopy // Jpn. J. Appl. Phys. 2008. V. 47. No. 3R. P. 1632-1634.

8. Zaytsev, K. I., Gavdush, A. A., et al. Highly accurate in vivo terahertz spectroscopy of healthy skin: variation of refractive index and absorption coefficient along the human body // IEEE Trans. THz Sci. Technol. 2015. V. 5. No. 5. P. $817-827$ 